\title{
Os determinantes da qualidade de vida em pessoas com HIV: uma revisão integrativa
}

\author{
Determinants of quality of life in people with HIV: an integrative review \\ Los determinantes de la calidad de vida en personas con VIH: una revisión integrativa
}

\author{
Jose Antonio Ponce Blandón'; Andrés González Bocanegrall; João Nunes Maidana Júnior"ll; \\ Diogo da Rosa Viana ${ }^{\prime V}$; María de las Mercedes Lomas Campos ${ }^{V}$
}

\begin{abstract}
RESUMO
Objetivo: analisar os determinantes que afetam a qualidade de vida de pessoas com HIV, a partir da revisão de pesquisas originais. Método: revisão integrativa da literatura com busca de produção sobre os determinantes da qualidade de vida de pacientes com HIV nas bases de dados PubMed, Scopus, CINAHL, CUIDEN e PsycINFO, realizada em abril de 2018. Resultados: foram identificadas 22 pesquisas, publicadas no período de 2012 a 2016. Detectou-se dispersão dos determinantes (42 variáveis) da qualidade de vida dessas pessoas. 0 apoio social $(36,3 \%)$, o estigma $(31,8 \%)$, a depressão $(31,8 \%)$ e a adesão $(22,7 \%)$ apareceram na revisão. Conclusão: existem múltiplos determinantes que afetam a qualidade de vida das pessoas com HIV, havendo consenso nos determinantes de apoio social, estigma, depressão e adesão.
\end{abstract} Descritores: Infecções por HIV; qualidade de vida; enfermagem; fatores epidemiológicos.

\section{ABSTRACT}

Objective: to examine the determinants affecting the quality of life of people with HIV by a review of original research. Method: this integrative literature review surveyed publications on determinants of quality of life in HIV patients drawn from the PubMed, Scopus, CINAHL, CUIDEN and PsycINFO databases during April 2018. Results: 22 studies published from 2012 to 2016 were identified. The review found the determinants of these people's quality of life to be dispersed ( 42 variables): social support appeared in $36.3 \%$, stigma in $31.8 \%$, depression in $31.8 \%$, and adherence in $22.7 \%$. Conclusion: multiple determinants affect the quality of life of people with HIV, and there is consensus that social support, stigma, depression and adherence are determinants.

Descriptors: HIV infections; quality of life; nursing; epidemiologic factors.

\section{RESUMEN}

Objetivo: analizar los determinantes que afectan la calidad de vida de las personas con VIH, a través de la revisión de investigaciones originales. Método: revisión bibliográfica integral, buscando producción sobre los determinantes de la calidad de vida en pacientes con VIH, en las bases de datos PubMed, Scopus, CINAHL, CUIDEN y PsycINFO, realizada durante abril de 2018. Resultados: um total de 22 artículos incluídos se incluyeron en la revisión. Se observó que existe una gran dispersión de determinantes relacionados a la calidad de vida (42 variables diferentes). El apoyo social (36,3\%), el estigma (31,8\%), la depresión $(31,8 \%)$ y la adhesión $(22,7 \%)$ son los que más frecuentemente aparecieron durante la revisión. Conclusión: existen múltiples determinantes que afectan a la calidad de vida de las personas con VIH, existiendo un consenso sobre los determinantes relativos al apoyo social, el estigma, la depresión y la adherencia.

Descriptores: Infecciones por VIH; calidad de vida; enfermería; factores epidemiológicos.

\section{INTRODUÇÃO}

Há uma tendência atualmente, ascendente no número de pessoas vivendo com o Vírus da Imunodeficiência Humana (HIV), com um aumento de mais de 8 milhões de pessoas em apenas 14 anos. Além de dizer que nesse mesmo ano, por causas relacionadas à Sindrome da imunodeficiência adquirida (AIDS), 1,2 milhão de pessoas morreram ${ }^{1}$.

A ficha apresentada pelo Programa Conjunto das Nações Unidas sobre HIV/AIDS (UNAIDS) em 2015 mostra uma queda de $42 \%$ nas mortes relacionadas à doença em comparação com 2004, ano em que o maior número de óbitos foi atingido ${ }^{2}$. Por um lado, o número de pessoas infectadas pelo HIV a cada ano continua a ser quantificado em milhões ${ }^{3}$. Neste sentido, a terapia antirretroviral não elimina completamente o HIV do organismo, obrigando o paciente a continuar o tratamento para o resto da vida ${ }^{4}$.

'Enfermeiro. Doutor em Enfermagem. Diretor e docente da Escola de Enfermagem da Cruz Vermelha Espanha. Universidade de Sevilha. Espanha. E-mail: japonce@cruzroja.es

"Enfermeiro. Mestrado em Ciências da Saúde. Escola de Enfermagem da Cruz Vermelha Espanha, Universidade de Sevilha. Espanha. E-mail: andres_agb94@hotmail.com I'Enfermeiro. Mestrado em Ciências da Saúde. Universidade Federal do Pampa. Uruguaiana RS, Brasil. E-mail: juniordana@hotmail.com

IVEnfermeiro. Mestrado em Ciências da Saúde. Universidade Federal do Pampa. Uruguaiana RS, Brasil. E-mail: diogoviana95@yahoo.com.br

vMédica. Doutora em Saúde Pública. Docente da Faculdade de Enfermagem, Fisioterapia e Podologia, Universidade de Sevilha. Espanha. E-mail: mlomas@us.es 
Nesta perspectiva, o tratamento atual do paciente com HIV só é efetivo se o paciente aderir ao tratamento antirretroviral. No entanto, estes medicamentos não são livres de efeitos adversos a curto e longo prazo ${ }^{5}$. Os efeitos adversos que o paciente experimenta não são apenas reduzidos aos produzidos pelo tratamento antirretroviral. Quando ele se torna um paciente crônico, ele experimenta uma séria deterioração emocional. Com isso, uma pessoa com HIV passa por uma série de mudanças na sua vida, relacionadas tanto com a medicação como com o próprio vírus, o que pode colocar sua qualidade de vida em risco.

Existe uma corrente atual que defende que o paciente é um ser holístico. Ao explorar a situação do paciente com HIV para este contexto atual, a necessidade de garantir que essa pessoa mantenha uma qualidade de vida aceitável torna-se fundamental. Logicamente, para poder intervir sobre a qualidade de vida das pessoas vivendo com o HIV, será necessário definir previamente quais são os fatores que afetam a qualidade de vida desses indivíduos e como eles podem agir sobre eles.

Podemos encontrar vários artigos de revisão que precedem este trabalho. Um exemplo é o apresentado em 2010, no qual o autor conclui que a relação entre a qualidade de vida do paciente com HIV e os indicadores sobre os parâmetros clínicos e a adesão ao tratamento não são muito consistentes. Além disso, o autor salienta que é essencial aprofundar no campo científico sobre a qualidade do cuidado e a gestão do diagnóstico e tratamento do HIV, devido à escassez de estudos encontrados ${ }^{6}$.

Portanto, esta revisão se torna relevante porque as pessoas com HIV apresentam prejuízos na qualidade de vida, mas há escassez de obras que a tratam de forma multidimensional e que coletem todas as variáveis que afetam a qualidade de vida. Esse fato dificulta a realização de intervenções que melhorem a qualidade de vida de pacientes soropositivos.

Foi observada a questão de investigação: Quais são os determinantes que afetam a qualidade de vida das pessoas com HIV? Dessa forma, este estudo teve por objetivo analisar os determinantes que afetam a qualidade de vida das pessoas com HIV, a partir da revisão de pesquisas originais.

\section{METOdOLOGIA}

Para a elaboração deste trabalho, foi realizada uma revisão de tipo integrativa, seguindo algumas das recomendações para a publicação de revisões sistemáticas ou metanálises do modelo Itens de Relatórios Preferidos para Revisões Sistemáticas e Meta-Análises (PRISMA) ${ }^{7}$.

Uma pesquisa de material bibliográfico publicado foi realizada em todo o mês de abril de 2018 . O motor de pesquisa da Biblioteca Nacional de Medicina dos Estados Unidos (PubMed), o mecanismo de pesquisa múltipla de bibliografia da editora Elsevier (Scopus) e o Cumulative Index to Nursing and Allied Health Literature (CINAHL) foram utilizados, devido à relevância que apresentam no panorama bibliográfico atual. Além disso, buscamos na base de dados bibliográfica da Fundação Index (CUIDEN), por ser um banco de dados específico de enfermagem, e na base de dados da American Psychological Association (PsycINFO), uma vez que é um motor de busca internacional específico para psicologia e sociologia (ramos de conhecimento que também se concentram na qualidade de vida das pessoas).

Em primeira instância, os descritores em Ciências da Saúde (DeCS) que poderiam facilitar o acesso a artigos relacionados sobre a qualidade de vida em pacientes com HIV, foram pesquisados no portal regional da Biblioteca Vitual em Saúde (BVS). Os seguintes descritores foram encontrados: HIV, Qualidade de vida e Criança. Além disso, o termo enfermagem adicionado para reduzir o levantamento apenas para documentos que tratam do assunto do campo de enfermagem. Os diferentes resultados obtidos foram analisados e os artigos foram selecionados com base nos seguintes critérios de inclusão: artigos publicados entre os anos de 2012 e 2018 para obter os resultados mais atuais possíveis, ter acesso aos textos completos, estudos realizados em população adulta e, por fim, artigos de pesquisa originais.

Foram excluídos os artigos que não contemplavam a enfermagem, as revisões bibliográficas, os estudos realizados em população de crianças, os artigos que apenas apresentaram o resumo e os artigos que tratassem da qualidade de vida dos pacientes com HIV e algum tipo de coinfecção, uma vez que a coinfecção poderia intervir nos resultados obtidos.

Foram obtidos 2.006 artigos no total. Depois de incluir o filtro da data (últimos 7 anos), os resultados foram reduzidos para 294 documentos, dos quais 8 foram repetidos e 5 não tiveram acesso ao texto completo. Dos 281 artigos resultantes, após a leitura dos títulos e resumos observou-se que apenas 22 concordaram com as características da revisão a serem realizadas ${ }^{8-29}$. Conforme mostra a figura 1 observa-se fluxograma com todo este processo. 

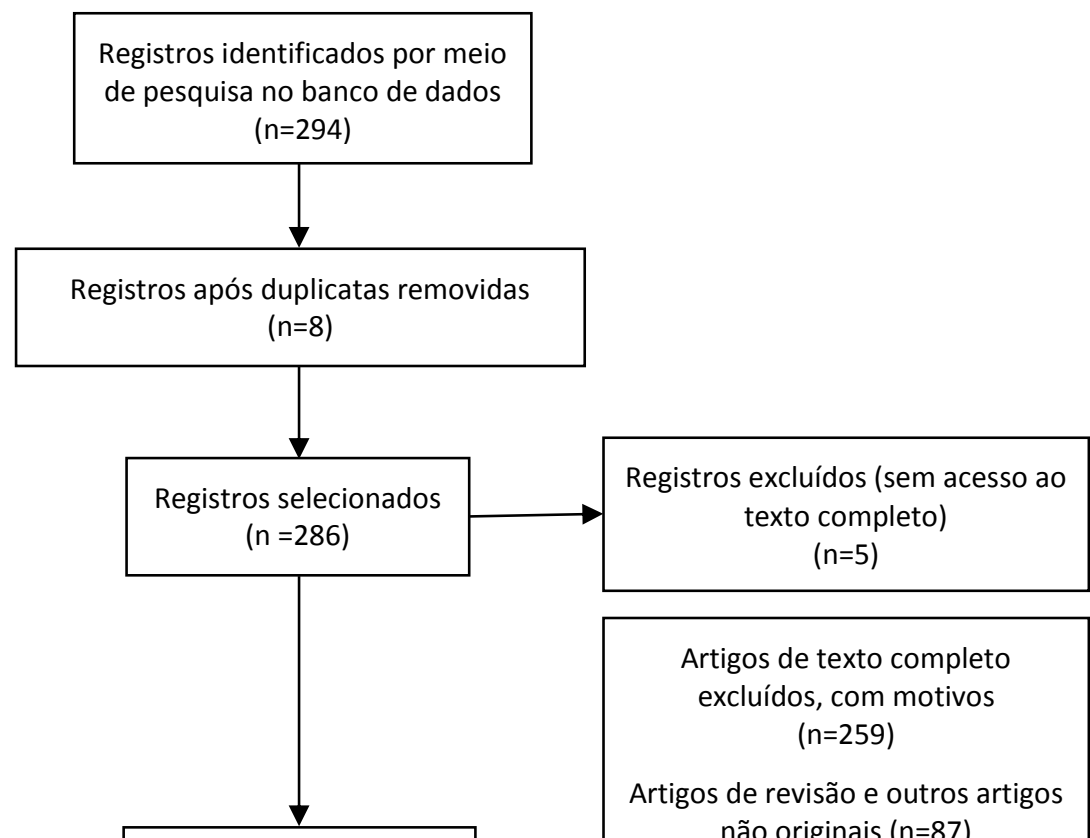

$$
(n=5)
$$

Artigos de texto completo excluídos, com motivos $(n=259)$

Artigos de revisão e outros artigos não originais $(n=87)$

Artigos que não estudaram a população adulta $(n=38)$

Artigos que não contemplavam a enfermagem $(n=98)$

Artigos que tratam a qualidade de vida dos pacientes com HIV e algum tipo de coinfecção
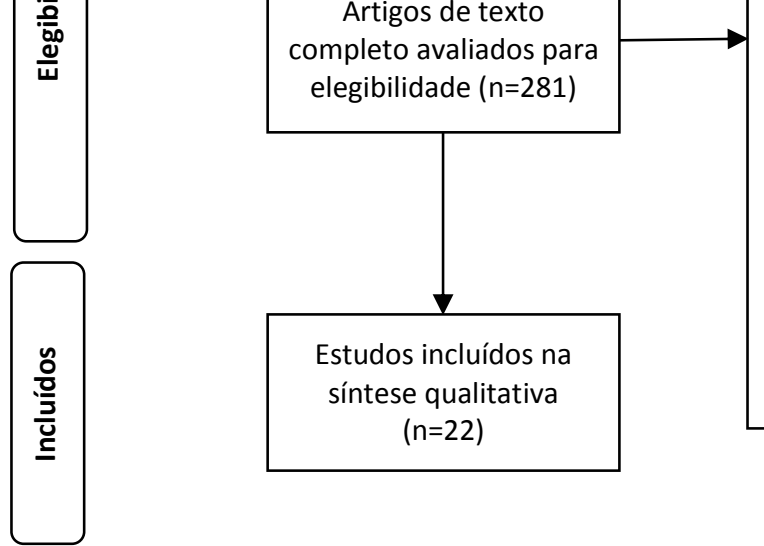

$(n=36)$

FIGURA 1: Fluxograma da revisão bibliografica

\section{RESULTADOS E DISCUSSÃO}

Foram analisados os 22 artigos que preencheram todos os critérios de seleção realizados ${ }^{8-29}$. Em todos os casos, o desenho utilizado foi de natureza quantitativa e descritiva, com exceção de um deles, que propôs uma metodologia qualitativa-quantitativa ${ }^{28}$. Existe uma alta frequência de estudos transversais, um total de 18 artigos que representam $81,8 \%$ de todos os documentos estudados $8,9,13-27,29$.

Com relação ao tamanho da amostra, observou-se uma média de $\mathrm{N}=238$ (DS=212), oscilando entre $34^{28}$ e $809^{11}$ indivíduos.

Em relação à proveniência, se observou que existe uma certa heterogeneidade. Mesmo assim, é necessário enfatizar que os países com maior peso no presente trabalho são: Brasil ${ }^{20,21,24-26,28,29}$, EUA ${ }^{10,15,18,19}$, China ${ }^{8,12,14,22}$ e Tailândia ${ }^{23,27}$, pois representam quase $80 \%$ da revisão.

Após uma análise detalhada de todos os artigos identificados, pode-se observar que o total das variáveis identificadas em todos eles somam 42 determinantes diferentes, que estão relacionados à qualidade de vida.

Muitos destes determinantes aparecem em mais de um dos 22 artigos selecionados. Este é o caso do determinante da qualidade de vida assistência social, que com uma aparição em 8 artigos (36,3\%) é o resultado que mais vezes foi identificado $0^{8,9,11-15,23}$. Com 7 artigos (31,8\%) segue-se depressão $0^{8,9,12,13,17,18,19}$ e estigma ${ }^{8,9,12,14-16,26}$. A adesão é outro determinate da qualidade de vida das pessoas vivendo com HIV que foi identificado em um total de 5 artigos $(22,7 \%)^{19-22,26}$. 
Outros determinantes que, embora em menor proporção, também apareceram nos trabalhos incluídos na presente revisão, seriam idade ${ }^{15,16,25,27}$, comorbidade ${ }^{15,18,28,29}$, educação ${ }^{16,17,23,24}$, renda ${ }^{23-26}$ e situação de trabalho ${ }^{23,25-27}$, em uma ordem de 4 artigos (18,2\%). O determinante de enfrentamento ${ }^{11,15,23}$ foi identificado como determinante da qualidade de vida de pessoas com HIV em 3 das obras (13,6\%). Quanto à raça ${ }^{24,25}$, síntomas ${ }^{10,18}$, eventos estresantes ${ }^{11,19}$, tratamento ${ }^{14,27}$, uso de drogas ${ }^{14,22}$, gênero ${ }^{16,24}$ e tempo decorrido desde o diagnóstico ${ }^{25,26}$, foram identificados como determinantes relacionados à qualidade de vida em apenas 2 artigos (9\%).

Os determinantes da qualidade de vida das pessoas soropositivas que apareceram somente em um artigo (4,5\%) são: evitação ${ }^{22}$, funcionamento familiar ${ }^{9}$, cuidados físicos ${ }^{9}$, permanência positiva ${ }^{9}$, níveis de CD4 ${ }^{14}$, estado civil ${ }^{16}$, percepção de controle da situação ${ }^{24}$, hábitos de vida saudáveis ${ }^{25}$, dor $^{17}$, mobilidade ${ }^{17}$, carga viral ${ }^{21}$, autoeficácia ${ }^{22}$, bemestar espiritual ${ }^{23}$, hospitalizações pela fase de $A_{I D S^{24}}$, mudanças corporais ${ }^{24}$, condição clínica ${ }^{24}$, autopercepção da doença ${ }^{24}$, atividade física ${ }^{25}$, nível socioeconômico ${ }^{25}$, conhecimento sobre a forma de infecção ${ }^{25}$, religiosidade ${ }^{26}$, sintomas psicossociais ${ }^{26}$, divulgação do estado sorológico ${ }^{27}$ e saúde geral ${ }^{27}$.

Conforme mostram as figuras 2 e 3, podem-se observar estes resultados principais de cada um dos artigos incluídos na revisão.

As limitações verificadas durante a condução deste estudo têm sido principalmente relacionadas à grande variabilidade dos determinantes encontrados. Isto é em grande parte devido à natureza multidimensional do conceito de qualidade de vida. A limitação teria sido salva ao ter focado a revisão em apenas um aspecto da qualidade de vida, mas nesse caso a visão global que o estudo queria oferecer teria sido perdida. A opção que poderia ser devido ao fato de que os determinantes relacionados à qualidade de vida das pessoas com HIV devem ser condicionadas à área sociodemográfica também deve ser avaliada.

Outra limitação que o estudo pode apresentar é que os resultados obtidos podem não ser totalmente confiáveis, uma vez que alguns determinantes são estudados por diferentes variáveis nos estudos analisados (por exemplo, a condição clínica é medida como tal em alguns estudos ${ }^{24}$ enquanto em outros é medida por níveis de CD $4^{14}$ ou através da carga viral apresentada pelo paciente ${ }^{21}$ ). Por conseguinte, seria conveniente analisar em estudos futuros a fiabilidade dos diferentes mecanismos existentes para determinar as variáveis relacionadas com a qualidade de vida.

Fazendo uma comparação dos resultados oferecidos por esta revisão e aqueles fornecidos por outros comentários anteriores, podemos observar como há uma certa dissonância. Em uma das mais antigas análises encontradas ${ }^{30}$, observamos que os determinantes que resultam são o acesso limitado à assistência médica por falta de recursos, estigma, problemas de saúde mental, uso de drogas e violência doméstica. Outro artigo do mesmo ano determina que os principais fatores associados à qualidade de vida são a fase de infecção em que a pessoa é encontrada, os parâmetros biológicos como a carga viral, CD4 e lipodistrofia. Posteriormente, encontramos outra revisão em que a adesão, religiosidade e espiritualidade, parâmetros clínicos, apoio social, comorbidade psiquiátrica e variáveis sociodemográficas são identificadas como determinantes ${ }^{6}$. Outros autores identificaram em seu estudo de revisão que os fatores mais relacionados à qualidade de vida são CD4, gênero e idade ${ }^{31}$. Finalmente, o artigo mais recente descobriu que uma revisão dos fatores relacionados à qualidade de vida dos pacientes com HIV determina que os mais frequentes e consistentemente associados são: emprego, estado imunológico, presença de sintomas, depressão, apoio social e adesão ${ }^{32}$, que coincide largamente com os quatro determinantes identificados por esta revisão como os mais relacionados à qualidade de vida (apoio social, depressão, estigma e aderência). A depressão explica até um $53 \%$ da variabilidade da qualidade de vida ${ }^{9}$, o apoio social até um $24 \%{ }^{11}$, o estigma até um $64 \%{ }^{15}$ e a adesão ao tratamento até um $27,2 \%^{19}$, segundo a bibliografia revisada. Esses números são consistentes com os achados encontrados em outros estudos similares que abordaram esses determinantes relacionados à qualidade de vida de pessoas com HIV, tanto na população geral ${ }^{30,32}$, quanto em grupos mais específicos, como mulheres em áreas rurais ${ }^{33}$.

Esses determinantes que aparecem mais intimamente relacionados à qualidade de vida, exigem que os profissionais que cuidam de pessoas com HIV, em especial os enfermeiros, tenham um perfil específico que facilite a compreensão da singularidade do processo de saúde pelo qual essas pessoas passam, com base na preparação de habilidades humanitárias e emocionais para abordar os determinantes que afetam a qualidade de vida das pessoas com HIV. Isso é reconhecido pelos profissionais de enfermagem que têm mais experiência em cuidar desse tipo de paciente ${ }^{34}$, embora também exijam um grande volume de protocolos de atenção, adequados às realidades regionais ou culturais específicas de cada localidade, que melhoram a motivação dos profissionais e a segurança no atendimento desses pacientes ${ }^{35}$.

No que se refere à discussão dos resultados obtidos, dizemos que foram identificados 42 determinantes que afetam a qualidade de vida das pessoas com HIV na totalidade da bibliografía revisada ${ }^{8-29}$, que são: permanecer positivo, bom enfrentamento, percepção da situação, autoeficácia, manter uma boa situação de trabalho e renda econômica, altos níveis socioeconômicos e educacionais, conhecimento sobre a forma de infecção pelo HIV, bem-estar espiritual, 
cuidados físicos, manutenção de uma boa condição clínica, saúde geral, baixos níveis de carga viral e altos níveis de CD4, hábitos de vida saudáveis, apoio social, bom funcionamento familiar, bom tratamento e adesão, ser mais velho, viver em uma família nuclear, ser uma pessoa religiosa, ser negro, gênero masculino, divulgar o estado serológico, mais tempo desde o diagnóstico, manter uma atitude de evitação, presença de comorbidade, internações por AIDS, o uso de drogas, depressão, sintomas psicossociais, estigma, autopercepção deficiente da doença, presença de eventos estressantes, sintomas experimentados, dor, dificuldade ou falta de mobilidade e a presença de alterações corporais.

\begin{tabular}{|c|c|c|c|}
\hline Autores & Local & Resultado & Conclusão \\
\hline $\begin{array}{l}\text { Li X, et al. } \\
2015^{8} \text {. }\end{array}$ & China & $\begin{array}{l}\text { A maior sintomatologia foi associada a maiores } \\
\text { níveis de estigma e menos apoio social. Os } \\
\text { sintomas depressivos também foram associados } \\
\text { com menor qualidade de vida. }\end{array}$ & $\begin{array}{l}\text { O estigma, o apoio social e os sintomas } \\
\text { depressivos são os principais fatores } \\
\text { associados à qualidade de vida em pessoas } \\
\text { com HIV. }\end{array}$ \\
\hline $\begin{array}{l}\text { Holtz C., et } \\
\text { al. } 2014^{9} \text {. }\end{array}$ & México & $\begin{array}{l}\text { Família, suporte emocional, cuidado físico e } \\
\text { permanência positiva foram positivamente } \\
\text { associados à qualidade de vida. Estigma, depressão } \\
\text { e evasão foram associados negativamente, e } \\
\text { explicam } 53 \% \text { da variabilidade. }\end{array}$ & $\begin{array}{l}\text { São necessários esforços para reduzir o } \\
\text { estigma e melhorar as redes de apoio às } \\
\text { pessoas com HIV, especialmente nas áreas } \\
\text { rurais. O estigma é uma barreira frequente } \\
\text { na busca de apoio à saúde. }\end{array}$ \\
\hline $\begin{array}{l}\text { Sousa HK., et } \\
\text { al. } 2014^{10} \text {. }\end{array}$ & EUA & $\begin{array}{l}\text { Uma variação substancial na variância da qualidade } \\
\text { de vida }(96,2 \%) \text { é explicada pela taxa de mudança } \\
\text { nos sintomas. }\end{array}$ & $\begin{array}{l}\text { O número de sintomas experimentados } \\
\text { tem uma forte associação com a qualidade } \\
\text { de vida. Uma gestão adequada dos } \\
\text { sintomas terá um grande benefício. }\end{array}$ \\
\hline $\begin{array}{l}\text { Nahas G., et } \\
\text { al. } 2015^{11} \text {. }\end{array}$ & Canadá & $\begin{array}{l}\text { O apoio social explica uma variação de } 24 \% \text { na } \\
\text { qualidade de vida. Relacionam-se à qualidade de } \\
\text { vida de forma significativa: suporte social, número } \\
\text { de eventos estressantes e sua intensidade. }\end{array}$ & $\begin{array}{l}\text { Os resultados sugerem que o apoio social } \\
\text { adequado tem um efeito mediador parcial } \\
\text { entre eventos estressantes e a qualidade } \\
\text { de vida dos pacientes vivendo com HIV. }\end{array}$ \\
\hline $\begin{array}{l}\text { Rao,D et al. } \\
2012^{12} \text {. }\end{array}$ & China & $\begin{array}{l}\text { Apoio social como mediador do estigma sobre } \\
\text { depressão e qualidade de vida. }\end{array}$ & $\begin{array}{l}\text { Melhorar o apoio social deve ser um } \\
\text { objetivo importante em intervenções } \\
\text { destinadas a reduzir o efeito do estigma na } \\
\text { saúde e na qualidade de vida. }\end{array}$ \\
\hline $\begin{array}{l}\text { Hou, W. et al. } \\
2014^{13} \text {. }\end{array}$ & Taiwan & $\begin{array}{l}\text { Os efeitos da depressão sobre a qualidade de vida } \\
\text { foram mitigados pela adição de suporte social, } \\
\text { embora permanecesse relevante. O apoio social foi } \\
\text { associado à qualidade de vida. }\end{array}$ & $\begin{array}{l}\text { O apoio social é um mediador parcial da } \\
\text { relação entre depressão e qualidade de } \\
\text { vida. }\end{array}$ \\
\hline $\begin{array}{l}\text { Wu, X. et al. } \\
2015^{14} \text {. }\end{array}$ & China & $\begin{array}{l}\text { O estigma percebido piora a qualidade de vida e o } \\
\text { suporte social melhora. Baixa pontuação de } \\
\text { estigma, alta pontuação de apoio contra o uso de } \\
\text { drogas e alta contagem de CD4 prevê uma variação } \\
\text { na qualidade de vida de } 49 \% \text {. }\end{array}$ & $\begin{array}{l}\text { O estigma percebido, suporte social, } \\
\text { contagem de CD4, estigma internalizado e } \\
\text { familiar, abuso de drogas e terapia } \\
\text { antirretroviral estão relacionados à } \\
\text { qualidade de vida de pacientes com HIV. }\end{array}$ \\
\hline $\begin{array}{l}\text { Slater, LZ et } \\
\text { al. } 2013^{15} \text {. }\end{array}$ & EUA & $\begin{array}{l}\text { Idade, apoio social e lidar com problemas foram } \\
\text { associados com melhor qualidade de vida. Estigma } \\
\text { social, enfrentamento centrado nas emoções } \\
\text { foram relacionados a pior qualidade de vida, e } \\
\text { representaram uma variância de } 64 \% \text {. }\end{array}$ & $\begin{array}{l}\text { Influência positiva do apoio social sobre a } \\
\text { qualidade de vida dos pacientes e um } \\
\text { efeito negativo das comorbidades médicas, } \\
\text { do estigma e da luta emocional. }\end{array}$ \\
\hline $\begin{array}{l}\text { Nazik, E. et } \\
\text { al. } 2013^{16} \text {. }\end{array}$ & Turquia & $\begin{array}{l}\text { O índice médio de qualidade de vida foi maior em } \\
\text { pacientes com idade entre } 36-45 \text { anos, mulheres e } \\
\text { pessoas de famílias nucleares. }\end{array}$ & $\begin{array}{l}\text { Seria aconselhável que famílias e } \\
\text { cuidadores recebessem algum tipo de } \\
\text { treinamento. Apoio e/ou educação sobre o } \\
\text { tratamento do ente querido que sofre de } \\
\text { uma doença estigmatizada. }\end{array}$ \\
\hline $\begin{array}{l}\text { Narsai P., et } \\
\text { al.2016 }\end{array}$ & $\begin{array}{l}\text { África } \\
\text { do Sul }\end{array}$ & $\begin{array}{l}\text { Aqueles que tinham um nível de educação superior } \\
\text { eram mais propensos a ter melhor qualidade de } \\
\text { vida e não experimentavam dor/desconforto. A } \\
\text { mobilidade está relacionada a uma melhor } \\
\text { qualidade de vida. }\end{array}$ & $\begin{array}{l}\text { São necessárias intervenções para reduzir a } \\
\text { prevalência de dor/desconforto e } \\
\text { aumentar a capacidade de participar das } \\
\text { atividades usuais, a fim de aumentar a } \\
\text { qualidade de vida das pessoas com HIV. }\end{array}$ \\
\hline $\begin{array}{l}\text { Gaynes, et al. } \\
2015^{18} \text {. }\end{array}$ & EUA & $\begin{array}{l}\text { Apenas } 18 \% \text { dos pacientes apresentaram } \\
\text { transtorno depressivo maior. As pessoas que } \\
\text { apresentaram comorbidade psiquiátrica relataram } \\
\text { pior saúde e qualidade de vida. }\end{array}$ & $\begin{array}{l}\text { Os transtornos depressivos estão } \\
\text { associados a uma maior gravidade da } \\
\text { doença e a uma deterioração da qualidade } \\
\text { de vida do paciente. }\end{array}$ \\
\hline
\end{tabular}

FIGURA 2: Características dos artigos revisados ${ }^{8-18}$ em abril de 2018. 


\begin{tabular}{|c|c|c|c|}
\hline Autores & Local & Resultado & Conclusão \\
\hline $\begin{array}{l}\text { Corless, IB. } \\
\text { et al. } 2013^{19} \text {. }\end{array}$ & EUA & $\begin{array}{l}\text { Eventos estressantes estão associados à adesão } \\
\text { do paciente ao tratamento. Forte relação entre } \\
\text { adesão ao tratamento e qualidade de vida, } \\
\text { explicando uma variação de } 27,2 \% \text { na qualidade } \\
\text { de vida. }\end{array}$ & $\begin{array}{l}\text { A avaliação rotineira das preocupações sobre } \\
\text { tratamento e eventos estressantes pode } \\
\text { promover uma melhor qualidade de vida e } \\
\text { uma melhor adesão }\end{array}$ \\
\hline $\begin{array}{l}\text { Galvão, } \\
\text { M.T., et al. } \\
2015^{20} \text {. }\end{array}$ & Brasil & $\begin{array}{l}\text { Houve uma relação significativa entre a adesão e } \\
\text { as dimensões da qualidade de vida relacionadas à } \\
\text { preocupação com a medicação e a confiança na } \\
\text { equipe que a dispensa. }\end{array}$ & $\begin{array}{l}\text { A adesão ao tratamento foi inadequada em } \\
\text { um grande número de sujeitos do estudo. } \\
\text { Quanto à qualidade de vida, isso foi } \\
\text { comprometido na maioria das dimensões da } \\
\text { escala. }\end{array}$ \\
\hline $\begin{array}{l}\text { Oliveira e } \\
\text { Silva, A.C., et } \\
\text { al. } 2014^{21} \text {. }\end{array}$ & Brasil & $\begin{array}{l}\text { A adesão e uma carga viral indetectável } \\
\text { melhoram a qualidade de vida. }\end{array}$ & $\begin{array}{l}\text { As pessoas aderentes têm melhor qualidade } \\
\text { de vida do que as que não o fazem. É } \\
\text { importante agir sobre a adesão dos pacientes } \\
\text { para melhorar sua qualidade de vida. }\end{array}$ \\
\hline $\begin{array}{l}\text { Huang L., et } \\
\text { al. } 2013^{22} \text {. }\end{array}$ & China & $\begin{array}{l}\text { A autoeficácia, a adesão e o uso de drogas foram } \\
\text { significativamente relacionados à qualidade de } \\
\text { vida. }\end{array}$ & $\begin{array}{l}\text { A autoeficácia no tratamento e a adesão à } \\
\text { medicação são preditores de qualidade de } \\
\text { vida. Se faz necessário realizar programas para } \\
\text { melhorar a autoeficácia dos pacientes. }\end{array}$ \\
\hline $\begin{array}{l}\text { Khumsaen } \\
\text { N., et al. } \\
2012^{23} \text {. }\end{array}$ & Tailândia & $\begin{array}{l}\text { Uma variação de } 42,7 \% \text { na qualidade de vida foi } \\
\text { relacionada ao nível de educação, renda mensal, } \\
\text { apoio social, bem-estar espiritual e } \\
\text { enfrentamento, adaptação, idade e vida com um } \\
\text { parente. }\end{array}$ & $\begin{array}{l}\text { Os programas para melhorar a qualidade de } \\
\text { vida devem se concentrar na promoção do } \\
\text { bem-estar espiritual. A incorporação de } \\
\text { sistemas de apoio social devem melhorar a } \\
\text { qualidade de vida. }\end{array}$ \\
\hline $\begin{array}{l}\text { Costa LT., et } \\
\text { al. } 2014^{24} \text {. }\end{array}$ & Brasil & $\begin{array}{l}\text { As variáveis que influenciam na qualidade de vida } \\
\text { são: gênero, educação, trabalho, renda, } \\
\text { hospitalizações por AIDS, alterações corporais, } \\
\text { condição clínica e autopercepção da doença. }\end{array}$ & $\begin{array}{l}\text { É importante promover a escuta ativa das } \\
\text { pessoas vivendo com HIV. Seria necessário } \\
\text { incluir o gerenciamento das variáveis dentro } \\
\text { da abordagem terapêutica do paciente com } \\
\text { HIV. }\end{array}$ \\
\hline $\begin{array}{l}\text { Pinto Okuno } \\
\text { M.F., et al. } \\
2015^{25} \text {. }\end{array}$ & Brasil & $\begin{array}{l}\text { As características relacionadas com maior } \\
\text { qualidade de vida são: atividade física, maior } \\
\text { tempo desde o diagnóstico, nível } \\
\text { socioeconômico, escolaridade, emprego, } \\
\text { conhecimento da forma de infecção e ser um } \\
\text { homem branco com menos de } 60 \text { anos. }\end{array}$ & $\begin{array}{l}\text { As orientações sobre formas de infecção, } \\
\text { tratamento e evolução da patologia, bem } \\
\text { como apoio social e psicológico, podem } \\
\text { aumentar a qualidade de vida das pessoas } \\
\text { com HIV. }\end{array}$ \\
\hline $\begin{array}{l}\text { Milanez } \\
\text { OFB., et al. } \\
2015^{26}\end{array}$ & Brasil & $\begin{array}{l}\text { Ocupação, renda, prática de uma religião, o } \\
\text { tempo decorrido desde o diagnóstico e a adesão } \\
\text { ao tratamento foram positivamente associados à } \\
\text { qualidade de vida. Foram negativamente } \\
\text { relacionadas: infecções oportunistas e relação } \\
\text { homoafetiva após ter sofrido. }\end{array}$ & $\begin{array}{l}\text { A qualidade de vida apresenta preditores } \\
\text { associados que foram identificados. Os } \\
\text { profissionais devem preferencialmente atuar } \\
\text { na redução do estigma e na redução dos } \\
\text { sintomas psicossociais. }\end{array}$ \\
\hline $\begin{array}{l}\text { Bunjoungma } \\
\text { nee } P \text {. } \\
2014^{27} \text {. }\end{array}$ & Tailândia & $\begin{array}{l}\text { A situação do trabalho é relacionada a qualidade } \\
\text { de vida da saúde física e mental. As pessoas que } \\
\text { divulgaram seu status serológico apresentaram } \\
\text { melhor qualidade de vida. }\end{array}$ & $\begin{array}{l}\text { Publicando o status serológico, ter uma boa } \\
\text { classificação geral de saúde por enfermagem e } \\
\text { ser empregado foram fatores preditivos } \\
\text { positivos para a saúde. }\end{array}$ \\
\hline $\begin{array}{l}\text { Lemos, LL. et } \\
\text { al. } 2012^{28} \text {. }\end{array}$ & Brasil & $\begin{array}{l}\text { A repercussão da coinfecção mostrou um } \\
\text { prejuízo para os domínios econômico, sexual e } \\
\text { furtivo da qualidade de vida. }\end{array}$ & $\begin{array}{l}\text { A existência de coinfecção causa mudanças na } \\
\text { qualidade de vida das pessoas com HIV. As } \\
\text { repercussões podem ser mitigadas com } \\
\text { intervenções que promovam a saúde. }\end{array}$ \\
\hline $\begin{array}{l}\text { Neves, LAS. } \\
\text { et al. } 2012^{29} \text {. }\end{array}$ & Brasil & $\begin{array}{l}\text { A qualidade de vida das pessoas com HIV que } \\
\text { tiveram coinfecção com tuberculose foi } \\
\text { diminuída em todos os campos de estudo. }\end{array}$ & $\begin{array}{l}\text { É necessário desenvolver estratégias } \\
\text { destinadas a reduzir o impacto epidemiológico } \\
\text { de uma doença, por outro. }\end{array}$ \\
\hline
\end{tabular}

FIGURA 3: Características dos artigos revisados ${ }^{19-29}$ em abril de 2018.

Seria necessário classificar as variáveis incluídas nos artigos da revisão de acordo com se o impacto sobre a qualidade de vida é positivo ou negativo. Assim, os determinantes que estão positivamente relacionados com a qualidade de vida são: manter-se positivo, bom enfrentamento, percepção da situação, autoeficácia, manter uma boa situação de trabalho e renda econômica, altos níveis socioeconômicos e educacionais, tendo conhecimento sobre o caminho de infecção por HIV, bem-estar espiritual, cuidados físicos, manutenção de uma boa condição clínica, saúde 
geral, baixos níveis de carga viral e altos níveis de CD4, hábitos de vida saudáveis, apoio social, bom funcionamento familiar, boa performance de tratamento e adesão, ser mais velho, viver em uma família nuclear, ser uma pessoa religiosa, ser negra, gênero masculino, divulgar o status serológico e mais tempo desde o diagnóstico.

Os determinantes que afetam negativamente a qualidade de vida são: manutenção de uma atitude de evitação, presença de comorbidade, hospitalizações por AIDS, uso de drogas, depressão, sintomas psicossociais, estigma, autopercepção deficiente da doença, presença de eventos estressantes, sintomatologia experimentada, dor, dificuldade ou falta de mobilidade e presença de alterações corporais.

Além disso, podemos classificar as variáveis identificadas como modificáveis e não modificáveis de acordo com a possibilidade de atuarem ou não ${ }^{11}$. Em total 5 dos 42 determinantes identificados (11,9\%) são considerados não modificáveis e são os seguintes: idade, raça, sexo, estado civil e religião. O restante das variáveis obtidas, em total 37, são variáveis modificáveis $(88,1 \%)$.

A ampla variabilidade das variáveis identificadas na revisão e a baixa coincidência entre os determinantes observados pelas diferentes revisões existentes indicam que ainda não está claro quais são os determinantes que

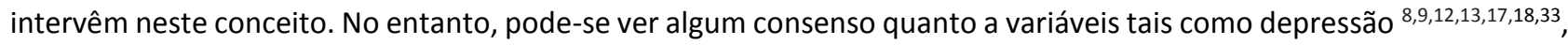
suporte social ${ }^{8,9,11-15,23}$, estigma ${ }^{8,9,12-14,20,33}$ e a adesão ${ }^{19-22,26}$.

\section{CONCLUSÃO}

A qualidade de vida é um conceito multidimensional que é influenciado por uma grande quantidade de fatores. Devido ao número de variáveis identificadas e à baixa coincidência entre elas, conclui-se que ainda não está claro quais são os determinantes que intervêm na qualidade de vida das pessoas com HIV.

De acordo com os resultados obtidos nesta revisão, os mais consensuais são: apoio social, depressão, estigma e aderência. É importante avaliar periodicamente as variáveis que afetam a qualidade de vida das pessoas com HIV para que os enfermeiros possam intervir melhorando sua qualidade de vida. Além disso, no plano social, seria conveniente criar programas que reduzam o estigma em relação a esse grupo de pessoas.

É necessário continuar investigando quais são os determinantes da qualidade de vida das pessoas com HIV, a fim de obter um conhecimento mais específico, consensual e atualizado sobre o assunto.

\section{REFERÊNCIAS}

1. WHO. Global Health Observatory data repository. [Internet] 2014 [citado em 15 fev 2018]. Avalaible from: http://apps.who.int/gho/data/view.main.22100?lang=en

2. UNAIDS. Fact sheet 2015. [cited 2018 feb 15]; Ginebra 27. Suiza. [Internet] 2015. Avalaible from: http://www.unaids.org/es/resources/campaigns/HowAIDSchangedeverything/factsheet

3. Ministerio de Sanidad, Servicios Sociales e Igualdad. Plan estratégico de prevención y control de la infección por el VIH y otras infecciones de transmisión sexual 2013-2016. [cited 2018 feb 27]; Madrid. [Internet] 2013. Avalaible from: https://www.mscbs.gob.es/ciudadanos/enfLesiones/enfTransmisibles/sida/docs/PlanEstrategico2013_2016.pdf

4. Ribera E, Tuset M, Martín M, Del Cacho E. Characteristics of antiretroviral drugs. Enferm. infecc. microbiol. clín. [Internet] 2011, [cited 2018 mar 3]; 29(5): 362-91. DOI: https://doi.org/10.1016/j.eimc.2011.02.004

5. Santos-Corraliza E, Fuertes-Martín A. Side effects of antiretroviral therapy. Fisiopathology, clinical manifestations and treatment. An. med. interna [Internet] 2006, [cited 2018 feb 22]; 23(7): 338-44. Avalaible from: http://scielo.isciii.es/scielo.php?script=sci_arttext\&pid=S0212-71992006000700010\&lng=es

6. Tavera M. Quality of life related to health in HIV patients. Rev. peru. epidemiol. (Online). [Internet] 2010, [cited 2018 mar 13]; 14(3): 7-14. Avalaible from: http://www.redalyc.org/pdf/2031/203119676002.pdf

7. Urrutia G, Bonfill X. PRISMA declaration: A proposal to improve the publication of systematic reviews and meta-analyses. Med. Clín. 2010; 135(11): 507-11. DOI: http://doi.org/10.1016/j.medcli.2010.01.015

8. Li X, Li L,Wang H, Fennie KP, Chen J, Williams AB. Mediation analysis of health-related quality of life among people living with HIV infection in China. Nurs. health sci. 2015; 17(2): 250-6. DOI: https://doi.org/10.1111/nhs.12181

9. Holtz C, Sowell R, VanBrackle L, Velasquez G, Hernandez-Alonso V. A Quantitative study of factors influencing quality of life in rural mexican women diagnosed with HIV. J. Assoc. Nurses AIDS Care. 2014; 25(6): 555-67. DOI: https://doi.org/10.1016/j.jana.2014.03.002

10. Sousa HK, Kwok O, Schmiege SJ, West SG. A longitudinal approach to understanding the relationship between symptom status and QOL. West j. nurs. res. 2014; 36(6): 732-47. DOI: https://doi.org/10.1177/0193945913510980

11. Nahas G, Côté J, Godin G, Otis J, Miranda J. Social support: Moderator or mediator effect on quality of life of persons living with HIV/AIDS? J. HIV AIDS soc. serv. 2015;14(3): 319-36. DOI: https://doi.org/10.1080/15381501.2014.919244

12. Rao D, Chen WT, Pearson CR, Simoni JM, Fredriksen-Goldsen K, Nelson K, et al. Social support mediates the relationship between HIV stigma and depression/quality of life among people living with HIV in Beijing, China. Int. j. STD AIDS. 2012; 23(7):481-4. DOI: http://doi.org/10.1258/ijsa.2009.009428 
13. Hou WL, Chen CE, Liu HY, Lai YY, Lee HC, Lee NY, et al. Mediating effects of social support on depression and quality of life among patients with HIV infection in Taiwan. AIDS care. 2014; 26(8): 996-1003. DOI:

https://doi.org/10.1080/09540121.2013.873764

14. Wu X, Chen J, Huang H, Liu Z, Li X, Wang H. Perceived stigma, medical social support and quality of life among people living with HIV/AIDS in Hunan, China. Appl. nurs. res. 2015; 28(2):169-74. DOI: http://doi.org/10.1016/j.apnr.2014.09.011

15. Slater LZ, Moneyham L, Vance DE, Raper JL, Mugavero MJ, Childs G. Support, stigma, health, coping, and quality of life in older gay men with HIV. J. Assoc. Nurses AIDS Care. 2013; 24(1): 38-49. DOI: http://doi.org/10.1016/j.jana.2012.02.006

16. Nazik E, Arslan S, Nazik H, Kurtaran B, Nazik S, Ulu A, et al. Determination of quality of life and their perceived social support from family of patients with HIV/AIDS. Sex. disabil. 2013; 31(3): 263-74. DOI: https://doi.org/10.1007/s11195-013-9304-x

17. Narsai P, Jinabhai CC, Taylor M, Stevens F. The health related quality of life of HIV positive respondents on antiretroviral treatment and HIV negative living in lower socio-economic housing in KwaZulu-Natal, South Africa. Soc. Indic. Res. 2016; 127(2): 851-63. DOI: https://doi.org/10.1007/s11205-015-0970-1

18. Gaynes BN, O'Donnell J, Nelson E, Heine A, Zinski A, Edwards M, et al. Psychiatric comorbidity in depressed HIV-infected individuals: common and clinically consequential. Gen. hosp. psychiatr. 2015; 37(4): 277-82. DOI: http://doi.org/10.1016/j.genhosppsych.2015.03.021

19. Corless IB, Voss J, Guarino AJ, Wantland D, Holzemer W, Jane Hamilton M, et al. The Impact of Stressful Life Events, Symptom Status, and Adherence Concerns on Quality of Life in People Living With HIV. J. Assoc. Nurses AIDS Care. 2013; 24(6): 478-90. DOI: http://doi.org/10.1016/j.jana.2012.11.005

20. Galvão MT, Soares LL, Pedrosa SC, Fiuza ML, Lemos LA. Quality of life and adherence to antiretroviral medication in people with HIV. Acta Paul. Enferm. 2015; 28(1):48-53. DOI: http://dx.doi.org/10.1590/1982-0194201500009

21. Oliveira e Silva AC, Karina RR, Almeida NJ, Gir E. Quality of life, clinical characteristics and treatment adherence of people living with HIV/AIDS. Rev. latinoam. enferm. 2014; 22(6): 994-1000. DOI: http://dx.doi.org/10.1590/0104-1169.3534.2508

22. Huang L, Li L, Zhang Y, Li H, Li X, Wang H. Self-efficacy, medication adherence, and quality of life among people living with HIV in human province of China: a questionnaire survey. J. Assoc. Nurses AIDS Care. 2013; 24(2):145-53. DOI: http://doi.org/10.1016/j.jana.2012.04.006

23. Khumsaen N, Aoup-por W, Thammachak P. Factors influencing quality of life among people living with HIV (PLWH) in Suphanburi province, Thailand. J. Assoc. Nurses AIDS Care. 2012; 23(1): 63-72. DOI: http://doi.org/10.1016/j.jana.2011.01.003

24. Costa LT, Cristina OD, Gomes TM, Formozo GA. Quality of life and people living with AIDS: relationship with sociodemographic and health aspects. Rev. latinoam. enferm. 2014; 22(4): 582-90. DOI: http://dx.doi.org/10.1590/0104-1169.3350.2455

25. Okuno MFP, Gosuen GC, Campanharo CRV, Fram DS, Batista REA, Belasco AGS. Quality of life, socioeconomic profile, knowledge and attitude toward sexuality from the perspectives of individuals living with Human Immunodeficiency Virus. Rev. latinoam. enferm. 2015; 23(2): 192-9. DOI: http://dx.doi.org/10.1590/0104-1169.3424.2542

26. Oliveira FB, Moura ME, Araújo TM, Andrade EM. Quality of life and associated factors in people living with HIV/AIDS. Acta Paul. Enferm. 2015; 28(6): 510-6. DOI: http://dx.doi.org/10.1590/1982-0194201500086

27. Bunjoungmanee $P$, Chunloy K, Tangsathapornpong A, Khawcharoenporn T, Apisarnthanarak A. Quality of life assessment among patients living with HIV/AIDS at a tertiary care hospital in Thailand. Southeast Asian j. trop. med. public health. [Internet] 2014, [cited 201825 apr]; 45(4):834-42. Avalaible from: https://www.tm.mahidol.ac.th/seameo/2014-45-4/08-5918-11.pdf

28. Lemos LA, Feijão AR, Gir E, Galvão MTG. Quality of life aspects of patients with HIV/tuberculosis co-infection. Acta Paul. Enferm. 2012; 25(1): 41-7. DOI: http://dx.doi.org/10.1590/S0103-21002012000800007

29. Neves LAS, Canini SEM, Reis RK, Santos CB, Gir E. Aids and tuberculosis: coinfection from the perspective of the quality of life of patients. Rev. Esc. Enferm. USP. 2012; 46(3):704-10. DOI: http://dx.doi.org/10.1590/S0080-62342012000300024

30. Aranda-Naranjo B. Quality of life in the HIV-positive patient: implications and consequences. J. int. assoc. provid. AIDS care 2004; 15 (Suppl. 5): 20S-27S. DOI: https://doi.org/10.1177/1055329004269183

31. Drewes J, Gusy B, Rüden U. More than 20 years of research into the quality of life of people with HIV and AIDS--a descriptive review of study characteristics and methodological approaches of published empirical studies. J. int. assoc. provid. AIDS care 2013; 12(1):18-22. DOI: https://doi.org/10.1177/1545109712456429

32. Degroote $S$, Vogalaers $D$, Vandijick DM. What determines health-related quality of life among people living with HIV: an updated review of the literature. Arch. public. health 2014; 72(40): 1-10. DOI: https://doi.org/10.1186/2049-3258-72-40

33. Vyavaharkar M, Moneyham L, Murdaugh C, Tavakoli A. Factors associated with quality of life among rural women with HIV disease. AIDS behav. 2012;16(2): 295-303. DOI: https://doi.org/10.1007/s10461-011-9917-y

34. Gomes AMT, Thiengo PCS, Oliveira DC, Rodrigues J. Social representation by health professionals of care for patients with HIV/AIDS. Rev. enferm. UERJ 2017; 25:e22321. DOI: https://doi.org/10.12957/reuerj.2017.22321

35. Araújo MCC, Acioli S, Neto M, Mello AS, Brandão PS. Nursing protocols: motivation and methodology in the shared construction process. Rev. enferm. UERJ 2017; 25:e27339. DOI: https://doi.org/10.12957/reuerj.2017.27339 